\title{
Coculture with hematopoietic stem cells protects cardiomyocytes against apoptosis via paracrine activation of AKT
}

Mark Rosenberg ${ }^{1 \dagger}$, Matthias Lutz $^{1 \dagger}$, Constantin Kühl ${ }^{1 \dagger}$, Rainer Will ${ }^{2}$, Volker Eckstein ${ }^{3}$, Jutta Krebs ${ }^{2}$, Hugo A Katus ${ }^{2}$ and Norbert Frey ${ }^{1 *}$

\begin{abstract}
Background: Previous experimental studies concluded that stem cells (SC) may exert their beneficial effects on the ischemic heart by paracrine activation of antiapoptotic pathways. In order to identify potential cardioprotective mediators, we performed a systematic analysis of the differential gene expression of hematopoietic SC after coculture with cardiomyocytes (CM).

Methods: After $48 \mathrm{~h}$ of coculture with neonatal rat ventricular CM (NRVCM), two consecutive cell sorting steps generated a highly purified population of conditioned murine hematopoietic SC (>99\%). Next, a genome-wide microarray analysis of cocultured vs. monocultured hematopoietic SC derived from three independent experiments was performed. The analysis of differentially expressed genes was focused on products that are secretable and/or membrane-bound and potentially involved in antiapoptotic signalling.

Results: We found CCL-12, Macrophage Inhibitory Factor, Fibronectin and connexin 40 significantly upregulated in our coculture model. An ELISA of cell culture supernatants was performed to confirm secretion of candidate genes and showed that coculture supernatants revealed markedly higher CCL-12 concentrations. Moreover, we stimulated NRVCM with concentrated coculture supernatants which resulted in a significant reduction of apoptosis compared to monoculture-derived supernatant. Mechanistically, NRVCMs stimulated with coculture supernatants showed a higher level of AKT-phosphorylation, consistent with enhanced antiapoptotic signaling.

Conclusion: In summary, our results show that the interaction between hematopoietic SC and NRVCM led to a modified gene expression and induction of antiapoptotic pathways. These findings may thus at least in part explain the cardioprotective effects of hematopoietic SC.
\end{abstract}

Keywords: Stem cells, Cardiomyocytes, Apoptosis, Paracrine

\section{Background}

Despite major advances in the treatment of coronary artery disease (CAD), acute myocardial infarction remains a major cause of death worldwide. In fact, the acute loss of blood supply potentially leads to apoptosis or necrosis of cardiomyocytes (CM) served by the infarct related artery resulting in ischemic cardiomyopathy and congestive

\footnotetext{
*Correspondence: norbert.frey@uksh.de

${ }^{\dagger}$ Equal contributors

'Department of Internal Medicine III (Cardiology and Angiology), University Medical Center Schleswig-Holstein, Campus Kiel, Schittenhelmstr.12, D-24105, Kiel, Germany

Full list of author information is available at the end of the article
}

heart failure. In the past the postmitotic heart had been considered a terminal differentiated organ unable to replace a significant loss of tissue such as that after an acute infarction [1]. This dogma has been challenged by the recent discovery of resident cardiac stem cells (SC) and the demonstration of hematopoietic SC that can home to the heart and transdifferentiate into cardiomyocytes $[2,3]$. These astonishing findings have led to the hypothesis that SC could be used for regeneration of infarcted myocardial tissue.

Therefore numerous studies have examined a potential therapeutic effect of bone marrow derived SC on myocardial function and regeneration after experimental

\section{Biomed Central}


myocardial infarction reviewed in [4]. Whereas some of these studies provided evidence for extensive myocardial regeneration after cellular cardiomyoplasty [5], others found no hematopoietic SC that had actually transdifferentiated into CM [6,7]. Yet, regardless of the variable effects on cardiac regeneration, virtually all of these studies found a significant improvement of cardiac function after cellular therapy. It is now generally accepted that SC therapy can favourably affect cardiac remodelling after myocardial infarction, although the scientific basis of this effect still remains to be elucidated.

Recent studies hypothesized that SC may exert their beneficial influence on cardiac repair by paracrine action on apoptosis or angiogenesis. It has been repeatedly shown that various SC types can produce and secrete a broad variety of cytokines, chemokines and growth factors that may be involved in cardiac repair [8-11].

On the basis of these experimental findings we postulated that the direct interaction of hematopoietic SCs with $\mathrm{CM}$ results in an upregulation of cardioprotective factors that can be secreted by hematopoietic SC and promote survival in cardiomyocytes. In order to identify interesting candidate genes, we performed a systematic analysis of the differential gene expression of hematopoietic SC after coculture with NRVCM with special emphasis on gene products involved in antiapoptotic signalling.

\section{Materials and methods Animals}

All experiments were performed in accordance with the Guide for the Care and Use of Laboratory Animals published by US National Institutes of Health. The animal study protocols were revised and approved by the Institutional Committee for the Ethics of Animal Care and Treatment.

\section{Isolation and culture of neonatal rat ventricular cardiomyocytes (NRVCM)}

1-2 days old Wistar rats (Charles River, Sulzfeld, Germany) were decapitized and hearts were harvested and minced in ADS. Subsequently, up to six digestion steps were carried out with pancreatin (Sigma, Munich, Germany, $0.6 \mathrm{mg} / \mathrm{ml}$ ) and collagenase type II (Worthington, $0.5 \mathrm{mg} / \mathrm{ml}$ ) in sterile ADS buffer containing $120 \mathrm{mmol} / \mathrm{l}$ $\mathrm{NaCl}, 20 \mathrm{mmol} / \mathrm{l}$ HEPES, $8 \mathrm{mmol} / \mathrm{l} \mathrm{NaH} 2 \mathrm{PO} 4,6 \mathrm{mmol} / \mathrm{l}$ glucose, $5 \mathrm{mmol} / \mathrm{l} \mathrm{KCl,} 0.8 \mathrm{mmol} / \mathrm{l} \mathrm{MgSO} 4, \mathrm{pH} 7.4$. NRVCM were purified from contaminating fibroblasts using a Percoll (Amersham, Germany) gradient centrifugation step. Finally, NRVCMs were resuspended and cultured in Dulbecco's modified Eagle's medium (DMEM) containing 10\% FCS, penicillin/streptomycin and L-glutamine (all from PAA, Linz, Austria) [12,13].
Isolation, purification and labelling of hematopoietic SC Isolation, purification and labelling of hematopoietic (lin-/c-kit+) SC were performed as described previously $[14,15]$ with the magnetic-activated cell sorting (MACS)kit from Miltenyi Biotech according to the manufacturer's protocol. Briefly, $\mathrm{C} 57 \mathrm{Bl} / 6$ mice were cervically dislocated. Shortly thereafter tibias and femurs were collected and flushed with phosphate-buffered solution (PBS) containing 2\% FCS. To remove cell clumps, crude bone marrow was filtered through a $30 \mu \mathrm{m}$ nylon mesh (Miltenyi Biotech). For the purpose of depleting mature blood cells such as T cells, B cells, monocytes/macrophages, granulocytes, erythrocytes as well as their commited precursors, bone marrow cells were incubated with a "cocktail" of biotinylated antibodies against a panel of "lineage" (lin) antigens, including CD5, CD45R (B220), CD11b, Anti-Gr-1 (Ly-6 G/C), 7-4 and Ter-119 (Lineage Cell Depletion Kit, Miltenyi Biotech). After addition of anti-biotin microbeads, lin-positive cells were separated using a magnetic column. Enrichment of the lin-negative cells for a subpool of cells expressing the SC marker c-kit/CD117 was performed by application of CD117 microbeads (Miltenyi Biotech). The purity of the separated cells was assessed by fluorescence-activated cell sorting (FACS) using a phycoerythrin labelled antibody against CD 117 (Pharmingen). Integrity and viability of purified lin-/c-kit + cells was confirmed by propidium iodide (PI) (Sigma) staining. In order to be able to detect and separate hematopoietic SC after culture with neonatal rat ventricular cardiac myocytes, lin-/c-kit + cells were labelled with the green fluorescent "cell tracker" carboxyfluorescein diacetate succinimydil ester (CFDA) (TefLabs). Lin-/c-kit + stem cells were therefore incubated for $30 \mathrm{~min}$ at $37^{\circ} \mathrm{C}$ with $5 \mu \mathrm{g} / \mathrm{ml}$ CFDA per $10^{6}$ cells. The staining process was concluded with two washing steps to assure clearance of any unbound CFDA.

\section{Coculture and separation of NRVCMs and Lin-/c-kit + stem cells}

The main objective of this study was to analyze the influence of a direct interaction with NRVCM on the gene expression profile of hematopoietic SC. Therefore, a coculture system of NRVCM with hematopoietic SC was established as follows: NRVCMs were isolated from whole hearts of 1-2 days old Wistar rats, resuspended and cultured at a density of $2 \times 10^{6}$ cells per well in an uncoated six well plate. After $48 \mathrm{~h}$, hematopoietic SC were separated from whole bone marrow of $\mathrm{C} 57 \mathrm{Bl} / 6$ mice and added at a density of $0.5 \times 10^{6}$ cells per well resulting in a ratio of NRVCM to lin-/c-kit + stem cells of 4:1. Cell culture conditions remained unchanged at $37^{\circ} \mathrm{C}$ and $5 \% \mathrm{CO}_{2}$ at all times. After $48 \mathrm{~h}$ the coculture was stopped and the supernatant removed, immediately 
frozen in liquid nitrogen and stored at $-80^{\circ} \mathrm{C}$ until further analysis. Next, remaining cells were washed twice with PBS and dissolved using a Trypsin-EDTA solution $(0.25 \%(\mathrm{w} / \mathrm{v})$, Invitrogen/GIBCO). Conditioned SC and NRVCM were then separated by two consecutive cell sorting steps using a FACS-Vantage SE flow cytometry system running CellQuest software (BD). Reliable discrimination of hematopoietic SC and NRVCM was ensured utilizing both CFDA staining and different forward-scatter and side-scatter signals. Viability and integrity of the cells was confirmed by staining with propidium iodide (Sigma).

In order to be able to test the importance of direct cellcell interaction between NRVCM and hematopoietic SC, conditioned cells were compared to identically treated lin-/c-kit + control cells that were held in a monoculture at a density of $2.5^{*} 10^{6}$ cells per well in uncoated six well plates. After $48 \mathrm{~h}$, cells were washed twice with PBS and dissolved using a Trypsin-EDTA solution $(0.25 \%(\mathrm{w} / \mathrm{v})$, Invitrogen/GIBCO). Monocultured SC were also sorted twice with FACS before being used for subsequent experiments. Viability and integrity of monocultured cells was again confirmed by staining with propidium iodide (Sigma).

Further processing of conditioned SC, NRVCM and supernatants is described in more detail in the Additional file 1: Methods section. This includes the experimental procedures of RNA isolation and purification, ELISA assays, the detection of apoptotic NRVCM as well as the stimulation of NRVCM with concentrated conditioned media and immunoblotting. Since most of the study results refer to analysis of the genetic profile of hematopoietic SC, we decided to describe the process of microarray hybridization and microarray data analyses as well as real-time PCR analyses of gene expression in more detail in the upcoming paragraphs.

\section{Probe labeling and Illumina sentrix BeadChip array hybridization}

Biotin-labeled cRNA samples for hybridization on Illumina Mouse Sentrix-6 BeadChip arrays (Illumina, Inc.) were prepared according to Illumina's recommended sample labeling procedure based on the modified Eberwine protocol [16]. In brief, $250 \mathrm{ng}$ total RNA was used for complementary DNA (cDNA) synthesis, followed by an amplification/labeling step (in vitro transcription) to synthesize biotin-labeled cRNA according to the MessageAmp II aRNA Amplification kit (Ambion, Inc., Austin, TX). Biotin-16-UTP was purchased from Roche Applied Science, Penzberg, Germany. The cRNA was column purified according to TotalPrep RNA Amplification Kit, and eluted in $60 \mu \mathrm{l}$ of water. cRNA Quality was checked using the RNA Nano Chip Assay on an
Agilent 2100 Bioanalyzer and spectrophotometrically quantified (NanoDrop).

Hybridization was performed at $58^{\circ} \mathrm{C}$ in GEX-HCB buffer (Illumina Inc.) at a concentration of $50 \mathrm{ng} \mathrm{cRNA} /$ $\mu \mathrm{l}$ and unsealed in a wet chamber for $20 \mathrm{~h}$. Spike-in controls for low, medium and highly abundant RNAs were added, as well as mismatch control and biotinylation control oligonucleotides. Microarrays were washed twice in E1BC buffer (Illumina) at room temperature for $5 \mathrm{~min}$. After blocking for $5 \mathrm{~min}$ in $4 \mathrm{ml}$ of $1 \%$ (wt/vol) Casein in phosphate buffered saline, Hammarsten grade (Pierce Biotechnology, Inc., Rockford, IL), array signals were developed by a 10-min incubation in $2 \mathrm{ml}$ of $1 \mu \mathrm{g} / \mathrm{ml}$ Cy3-streptavidin (Amersham Biosciences, Buckinghamshire, UK) solution and 1\% blocking solution. After a final wash in E1BC, the arrays were dried and scanned.

\section{Scanning and data analysis}

Microarray scanning was done using a Beadstation array scanner. Settings were adjusted to a scaling factor of 1 and PMT settings at 430. Data extraction was done for all beads individually, and outliers were removed when MAD (median absolute deviation) was $>2.5$. All remaining data points were used for the calculation of the mean average signal for a given probe, and the standard deviation for each probe was calculated. Array data were normalized using a quartile-normalization algorithm. To select differentially expressed trancripts, an empirical Bayes analysis was carried out. Transcripts were selected as differentially expressed with $\mathrm{p}$-values $<0.05$ and corrected for multiple testing by a Benjamini \& Hochberg algorithm [17]. Gene expression was visualized by scatterplots and heatmaps. Data analysis was performed with $\mathrm{R}$ (version 2.7.2) and Bioconductor (version 2.0.1) using the packages beadarray (version 1.8.0) and limma (version 2.14.7). Differentially expressed transcripts were further classified by cellular location of the corresponding proteins using the LOCATE subcellular localization database (http://locate.imb.uq.edu.au/) and according to their function using PANTHER Classification System (http://www.pantherdb.org/).

\section{Quantitative real-time PCR analysis of gene expression}

Real-time PCR (RT-PCR) was used to further validate data generated in the microarray analysis and performed as follows: $100 \mathrm{ng}$ of DNase-digested total RNA of each condition was transcribed to first strand cDNA with the Transcriptor first strand cDNA synthesis kit (Roche, Germany). Reaction conditions were set as recommended by the supplier. Real time PCR primers (MWG Biotech) were designed assisted by the Primer 3 software (http://primer3.sourceforge.net/). Targets were normalized using oligonucleotide primers for GAPDH as an internal standard. The resulting amplicons contained 
an exon-intron-exon boundary. The ABI Prism 7700 Sequence Detection System (Perkin Elmer Applied Biosystems) and the Platinum SYBR Green qPCR SuperMix-UDG (Invitrogen) were used for performing real-time PCR from reverse transcribed cDNA samples following the manufacturer's instructions. Specificity of the reactions was checked by melting curve analysis and by verifying the correct size of the product on a $2 \%$ agarose gel. Each PCR amplification was carried out in duplicate wells, using the following conditions: $2 \mathrm{~min}$ at $50^{\circ} \mathrm{C}, 2 \mathrm{~min}$ at $95^{\circ} \mathrm{C}$, followed by a total of 40 temperature cycles $\left(15 \mathrm{~s}\right.$ at $95^{\circ} \mathrm{C}$ and $30 \mathrm{~s}$ at $\left.60^{\circ} \mathrm{C}\right)$.

\section{Statistical analysis}

Data are presented as mean \pm SEM. Statistical analysis was carried out with Students- $t$-Test, if not noted differently. $P<0.05$ was considered statistically significant.

\section{Results}

\section{Generation of a highly purified population of} hematopoietic SC after coculture with NRVCM

Hematopoietic SC were isolated from male C57BL/6 mice by magnetic cell sorting, typically resulting in a yield of 1.1-1.4 x $10^{8}$ bone marrow cells per dissected mouse. After the separation process, approximately $0.9-1.2 \times 10^{6}$ hematopoietic SC were obtained, corresponding to $\sim 1 \%$ of all isolated bone marrow cells. FACS-analyses were performed to check for purity and viability of the separated stem cells. NRVCM were prepared from 1-2 days old Wistar Rats using a percoll gradient centrifugation.

In order to elucidate potential molecular mechanisms of SC mediated cardiac regeneration and repair, we established a coculture system of CFDA labeled hematopoietic SC and NRVCM at a ratio of 1:4 per well (Figure 1). After $48 \mathrm{~h}$ of coculture, cells were separated by FACS (Figure 2A). Two consecutive cell-sorting steps enabled us to generate a highly purified (purity $>99.5 \%$ ) population of hematopoietic SC that were stimulated by NRVCM for $48 \mathrm{~h}$ (Figure 2B). These cells were subsequently used for further microarray analyses and compared to identically treated hematopoietic SC that were held in a monoculture for $48 \mathrm{~h}$.

\section{Microarray analysis of hematopoietic SC after coculture with NRVCM}

We hypothesized that the direct interaction of hematopoietic SC with NRVCM may lead to an upregulation of cardioprotective genes in SC. Therefore we performed a genome-wide transcriptome microarray analysis of cocultured versus monocultured hematopoietic SC. We used RNA of three independent coculture and three monoculture experiments, respectively. Bayes analysis of the raw data revealed 3014 differentially regulated transcripts. Genes were considered differentially regulated, when up- or downregulation exceeded 50\%. Regulation status in the scatter blot is colour coded. Upregulated genes appear red, downregulated genes blue and genes

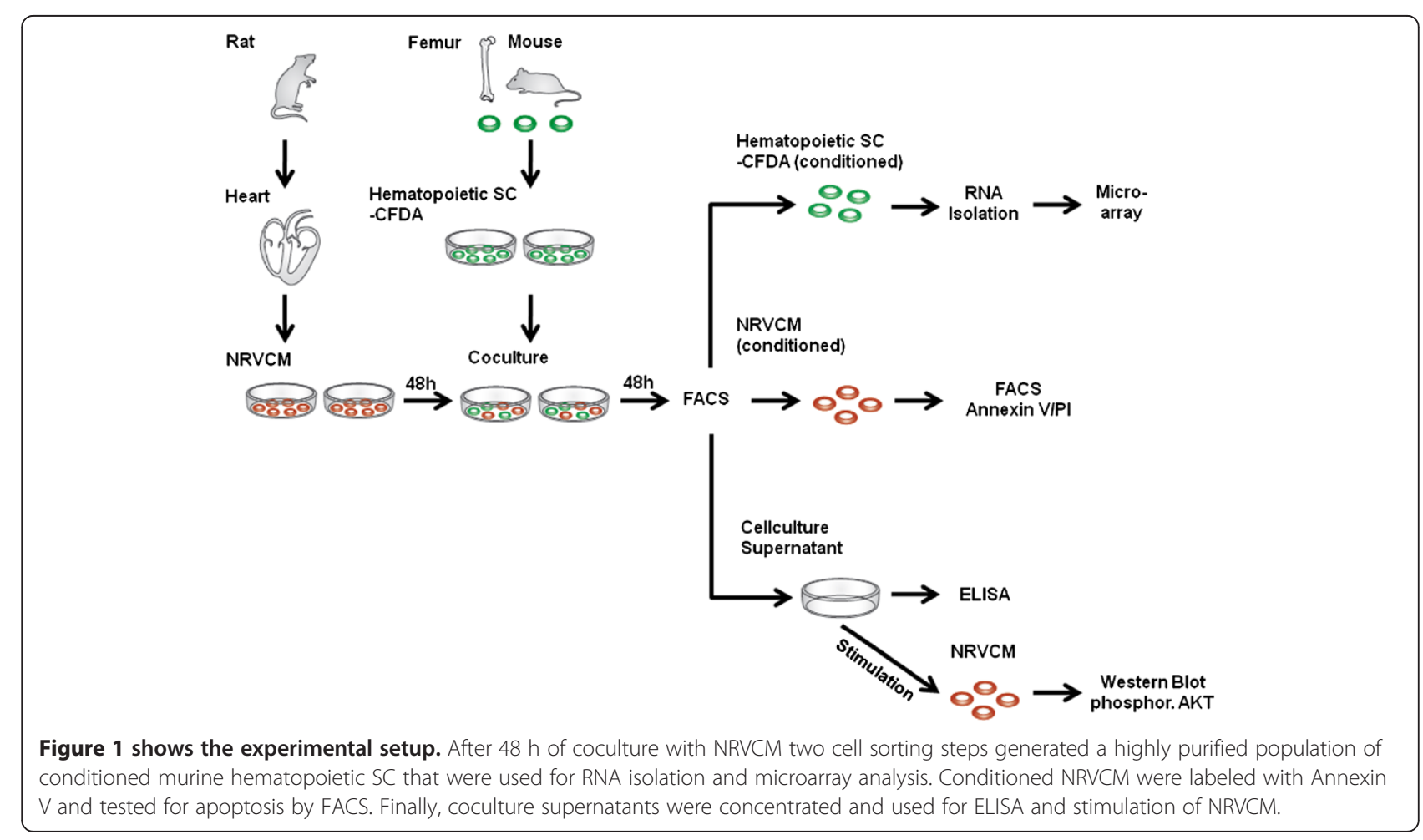



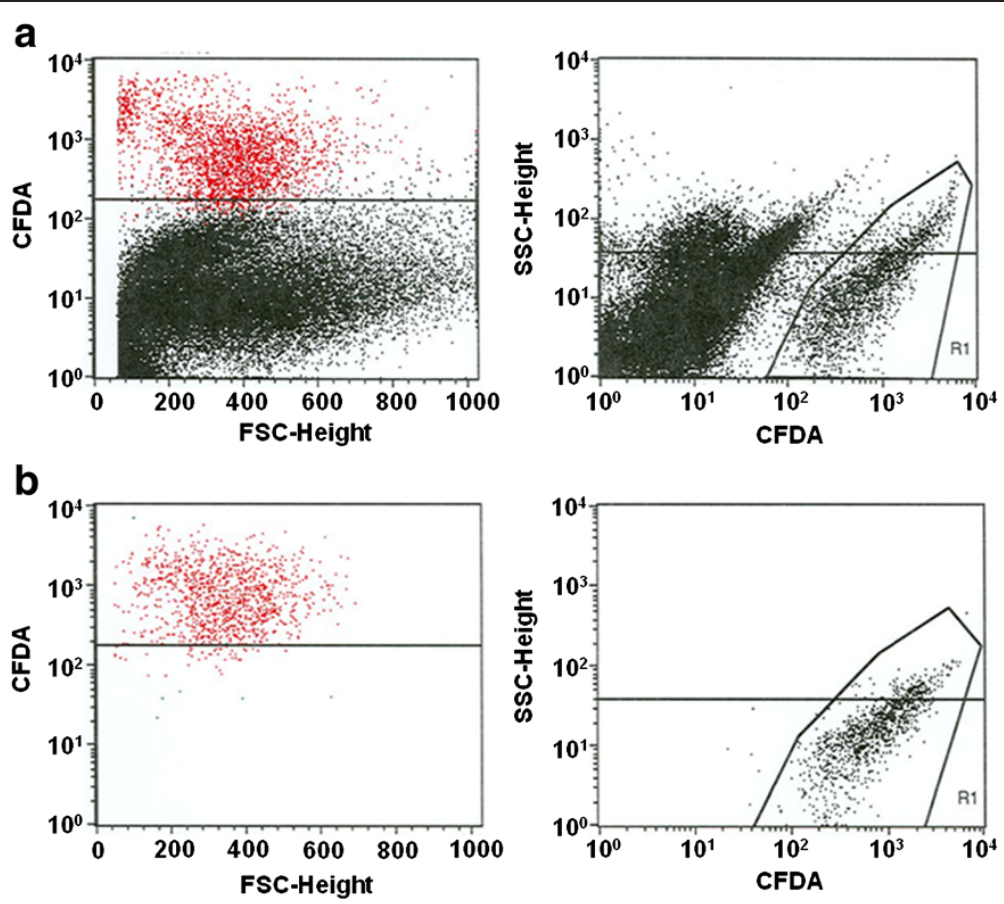

Figure 2 illustrates the FACS analysis of CFDA labeled cocultured hematopoietic SC. (a) Here we show the coculture prior to separation. After two consecutive cell sorting steps a highly purified population of hematopoietic SC with a purity $>99.5 \%$ were generated and used for further experiments (b).

with no differential regulation are represented by black dots. 1500 were significantly $(\mathrm{p}<0.05)$ and more than 1.5 fold upregulated (Figure 3A), whereas 1514 were significantly downregulated (less than 0.5 fold). Next, resulting data were normalized and displayed in heat maps, revealing highly reproducible results within groups and a low variance between microarray hybridizations (Figure 3B). We hypothesized that the close interaction of hematopoietic SC with NRVCM may lead to differential regulation of genes that promote survival of NRVCM via inhibition of apoptosis. Therefore, further processing of data generated from the microarray analysis focused on upregulated genes with secretable products that interfere with apoptotic pathways. We used two different databases (LOCATE, PANTHER) to identify candidate genes that met these criteria. Thereby, we were able to restrict our list to 8 genes. Specifically, CXCL 1, CCL 6, CCL 12, EGFL 7, FN 1, GJA 5, MIF and TIMP were shown to be not only significantly upregulated in our microarray analysis but also fulfilled our above mentioned prerequisites.
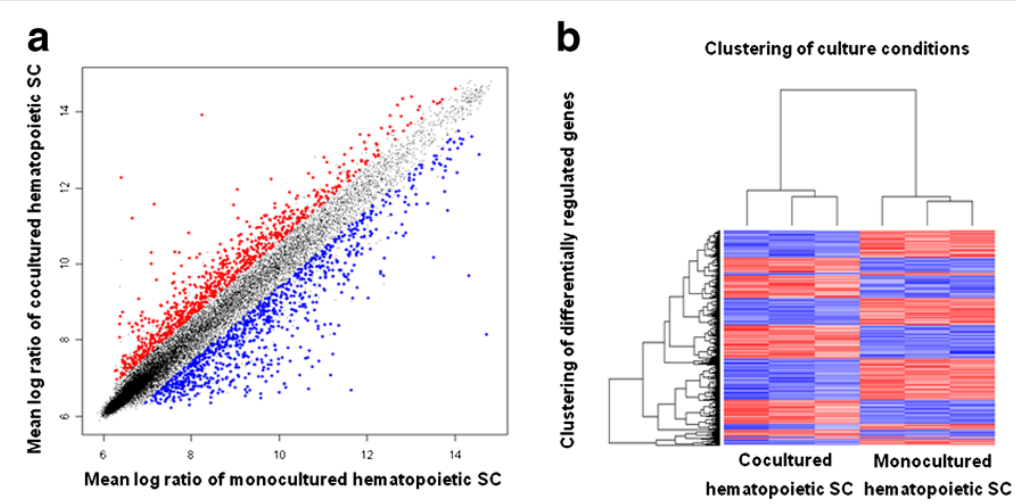

Figure 3 displays the microarray analysis of cocultured hematopoietic SC. (a) Scatter plots showing the log ratios of the means of differentially regulated transcripts in cocultured hematopoietic SC plotted against monocultured hematopoietic SC. Red dots represent upregulation, blue dots downregulation. (b) Heat map displaying 3014 differentially regulated transcripts. Red colour represents upregulation, blue colour downregulation. 
A more detailed list is provided in table 1 . The data discussed in this publication have been deposited in NCBI's Gene Expression Omnibus [18] and are accessible through GEO Series accession number GSE21098 (http://www. ncbi.nlm.nih.gov/geo/query/acc.cgi? token = vvyhramgioykypi\&acc = GSE21098). Interestingly, we found no evidence for any transdifferentiation of hematopoietic SC towards a cardiac lineage. Consistently, early cardiacspecific transcripts like Nkx2-5, MEF2C, GATA4, markers of the primary heart field like Tbox 5 and Hand 1 or secondary heart field such as Isl1 and Fgf10, were not found to be differentially expressed, neither were later cardiac specific transcripts like alpha-MHC, Troponin-T, alphaactinin, and Troponin-I. Flk-1, which is known to be a marker for multilineage mesoderm progenitor cells, was not differentially expressed as well. In addition, these findings confirm the high purity of our cell separation, resulting in no measurable contamination of hematopoietic SC with NRVCM RNA.

Moreover, our number of differentially regulated genes in the microarray analysis was rather high. We have therefore added a list of genes that had an adjusted p-value of $<0.05$ and an upregulation of more than 1.5-fold. Next, we used DAVID Bioinformatics Ressources v6.7 to functionally annotate our transcripts via Gene Ontology $[19,20]$. We selected transcripts with the following Gene Ontology terms for further analysis: Apoptosis, Angiogenesis, Proliferation, Heart development, Immune response, Cell migration, Cell growth. These genes were classified according to their biological function. This list is added to the manuscript as Additional file 2: Table S1.

\section{Confirmation of microarray data by real-time PCR}

We used quantitative Real-Time PCR to verify differential expression of selected genes in cocultured and monocultured hematopoietic SC. We primarily focused on genes with potentially secretable and antiapoptotic products for which an upregulation was predicted by our microarray

\section{Table 1 Differentially regulated antiapoptotic genes in cocultured hematopoietic SC detected by microarray analyses}

\begin{tabular}{llll}
\hline Symbol Gene-Name & \multicolumn{2}{l}{ Fold-Change $\boldsymbol{p}$-Value } \\
\hline CCL6 & Chemokine (C-C motif) ligand 6 & 3.04 & 0.01 \\
\hline CCL12 & Chemokine (C-C motif) ligand 12 & 4.65 & 0.029 \\
\hline CXCL1 & Chemokine (C-X-C motif) ligand 1 & 4.42 & 0.049 \\
\hline EGFL7 & EGF-like domain 7, transcript variant C & 4.18 & 0.002 \\
\hline FN1 & Fibronectin 1 & 5.16 & 0.017 \\
\hline GJA5 & $\begin{array}{l}\text { Gap Junction membrane } \\
\text { channel protein alpha 5 }\end{array}$ & 1.57 & 0.001 \\
\hline MIF & Macrophage migration inhibitory factor & \\
\hline TIMP & Tissue inhibitor of metalloproteinase & 1.44 & 0.004 \\
\hline
\end{tabular}

data. Thereby we were able to show that coculture of hematopoietic SC with NRVCM for $48 \mathrm{~h}$ leads to an 14.8 fold upregulation of the chemokine (C-C motif) ligand 12 (CCL12) $(p<0.05)$, whereas expression of the gap junction membrane channel protein alpha 5 (GJA5, Connexin 40) was 4.5 fold induced ( $p<0.05$ versus control). Furthermore, real-time data revealed a significant 1.8 fold upregulation of the macrophage migration inhibitory factor and a 5.6-fold overexpression of Fibronectin in conditioned hematopoietic SC $(p<0.001)$ (Figure 4A). In contrast, we could not confirm significant differential expression of CXCL1, CCL 6, EGFL 7 or TIMP by real-time PCR, although the trend of their differential expression was consistent with the microarray data $(\mathrm{p}>0.05)$.

\section{Antiapoptotic proteins are elevated in supernatants of cocultured hematopoietic SC}

We initially hypothesized that cocultured hematopoietic SC express antiapoptotic proteins that may exert beneficial effects on NRVCM survival via a paracrine mechanism. We therefore collected and analyzed supernatants of several coculture experiments for increased levels of proteins identified in the microarray experiments. We measured the concentration of CCL12 in concentrated supernatants of three independent mono- and coculture experiments by ELISA. Thereby we were able to show that the CCL12 concentration is indeed significantly elevated in concentrated supernatants collected under coculture conditions when compared to monoculture experiments $(21 \mathrm{pg} / \mathrm{ml}$ vs. $3,3 \mathrm{pg} / \mathrm{ml} ; p<0.05)$ (Figure $4 \mathrm{~B}$ ).

\section{Coculture with hematopoietic SC leads to inhibition of NRVCM apoptosis}

Since our findings demonstrated that coculture of hematopoietic SC with NRVCM leads to an overexpression of secretable antiapoptotic proteins in SC, we were next asking whether these findings also translated into a reduced apoptosis rate in cocultured NRVCM. In order to address this question we repeated our coculture experiments with PI-/Annexin-V-staining. After $48 \mathrm{~h}$ of coculture and monoculture, we used APC-conjugated Annexin- $\mathrm{V}$ and PI to identify the fraction of apoptotic cells (Annexin $\mathrm{V}$ positive, PI negative) within the population of cocultured NRVCM (CFDA-/APC+/PI-) in comparison to identically stained NRVCM in monoculture. Indeed, coculture with hematopoietic SC cuts the number of apoptotic NRVCM by half when compared to monocultured NRVCM ( $12 \%$ vs. $24 \% ; p<0.05 ; \mathrm{n}=5)$ (Figure 5).

\section{Antiapoptotic effects are mediated via activation of the AKT/PKB pathway}

Finally, we sought to begin to investigate the underlying molecular mechanism that mediates antiapoptotic signals 

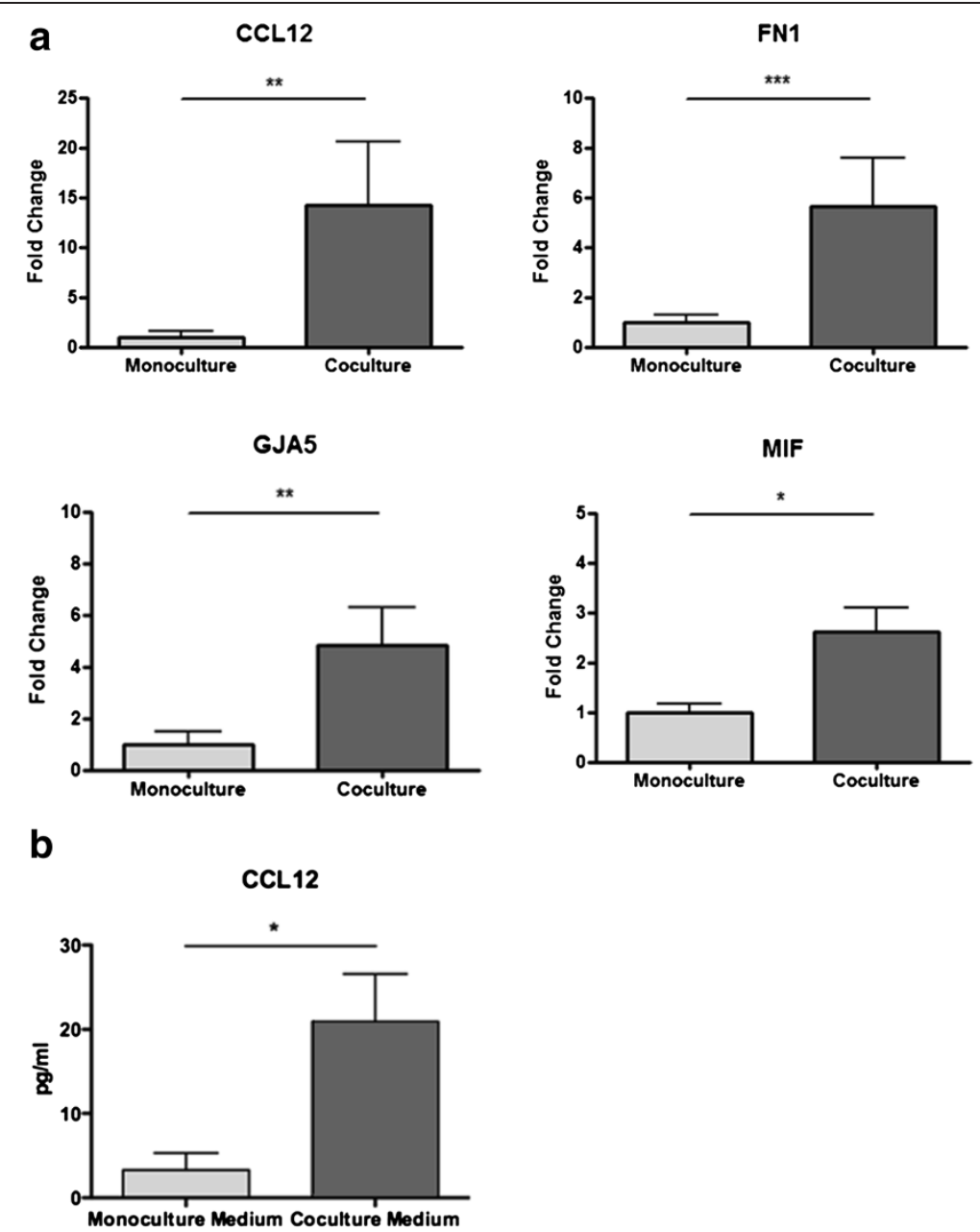

Figure 4 demonstrates that coculture with NRVCM leads to overexpression of antiapoptotic factors in hematopoietic SC. (a) CCL-12, FN1, GJA-5 and MIF mRNAs are upregulated in cocultured hematopoietic SC $(n=8)$. Levels of significance ${ }^{*} p<0.05,{ }^{* *} p<0.01,{ }^{* * *} p<0.001$. (b) ELISA from concentrated cell culture supernatants show higher CCL-12 levels in coculture supernatants compared to monoculture supernatants $(n=3)$. Level of significance ${ }^{*} p<0.05$.

in cocultured NRVCM. In this context, it has been shown before that CCL 12, FN-1 and MIF can trigger phosphorylation of the antiapoptotic protein AKT via activation of PI3K [21-23]. We therefore tested the hypothesis that inhibition of apoptosis in cocultured NRVCM is related to an increased phosphorylation of AKT induced by factors secreted from hematopoietic SC in a paracrine manner. Hence, we used concentrated cell culture supernatants of several NRVCM/hematopoietic SC coculture and hematopoietic SC monoculture experiments to stimulate NRVCM that were held in a monoculture for $48 \mathrm{~h}$. Densitometry of western-blots was utilized to compare the activation of selected signal transduction cascades. We found the anti-apoptotic AKT/PKB-pathway being significantly higher activated (ratio of PhospoAkt473 to Akt) after stimulation with coculture medium compared to monoculture medium (5.2 vs. 3.2 arbitrary units, $p<0.01$ ) (Figure 6). These findings suggest that survival of NRVCM that are held in a coculture with hematopoietic SC is improved by paracrine activation of the AKT/PKB signal transduction cascade.

\section{Discussion}

Despite the fascinating idea of myocardial regeneration by undifferentiated precursor cells, the observed clinical effects of cellular cardiomyoplasty so far are rather modest and the underlying molecular mechanisms still remain unclear. One of the proposed mechanisms is the secretion of paracrine factors by SC that can modify cardioprotective signaling. The identification of these cytokines would be of high clinical relevance, since it may offer the possibility to establish new therapeutic options after myocardial infarction.

We thus hypothesized that a systematic analysis of the differential gene expression of hematopoietic SC after coculture with NRVCM may lead to the recognition 

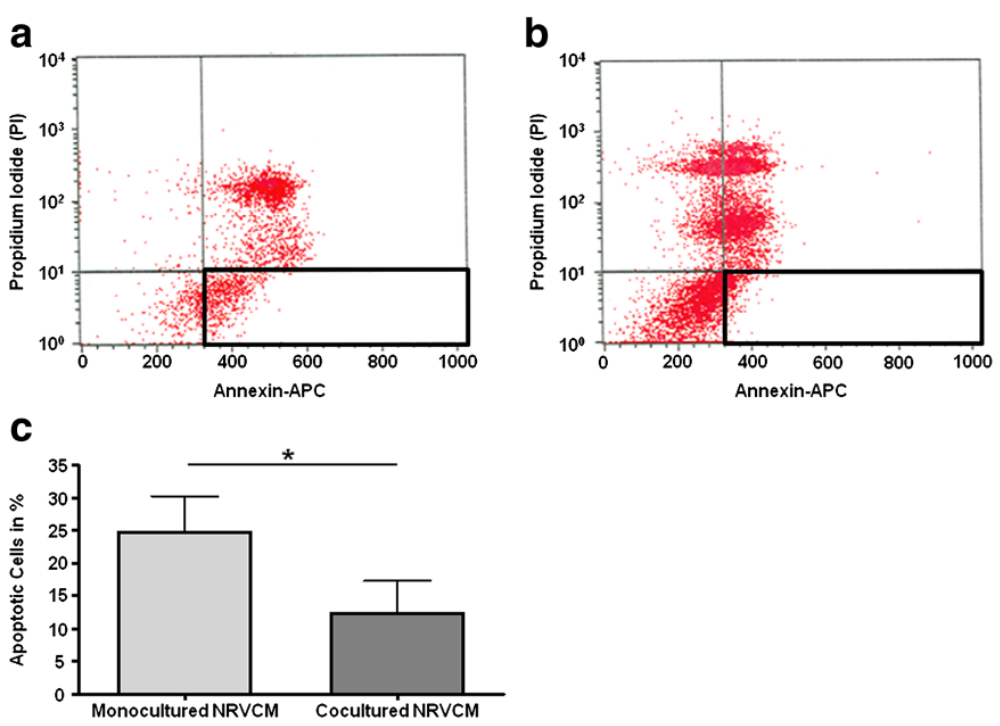

Figure 5 confirms that coculture with hematopoietic SC inhibits apoptosis in NRVCM. NRVCM were stained with Annexin-V-/ Propidiumiodid and analyzed for apoptosis after coculture (a) or monoculture (b). Analysis of five experiments reveal significant less apoptotis in cocultured NRVCM (Annexin-V-positive, Pl-negative cells) (c). All values are expressed as means +/- SEM. Level of significance: ${ }^{*} p<0.05$.

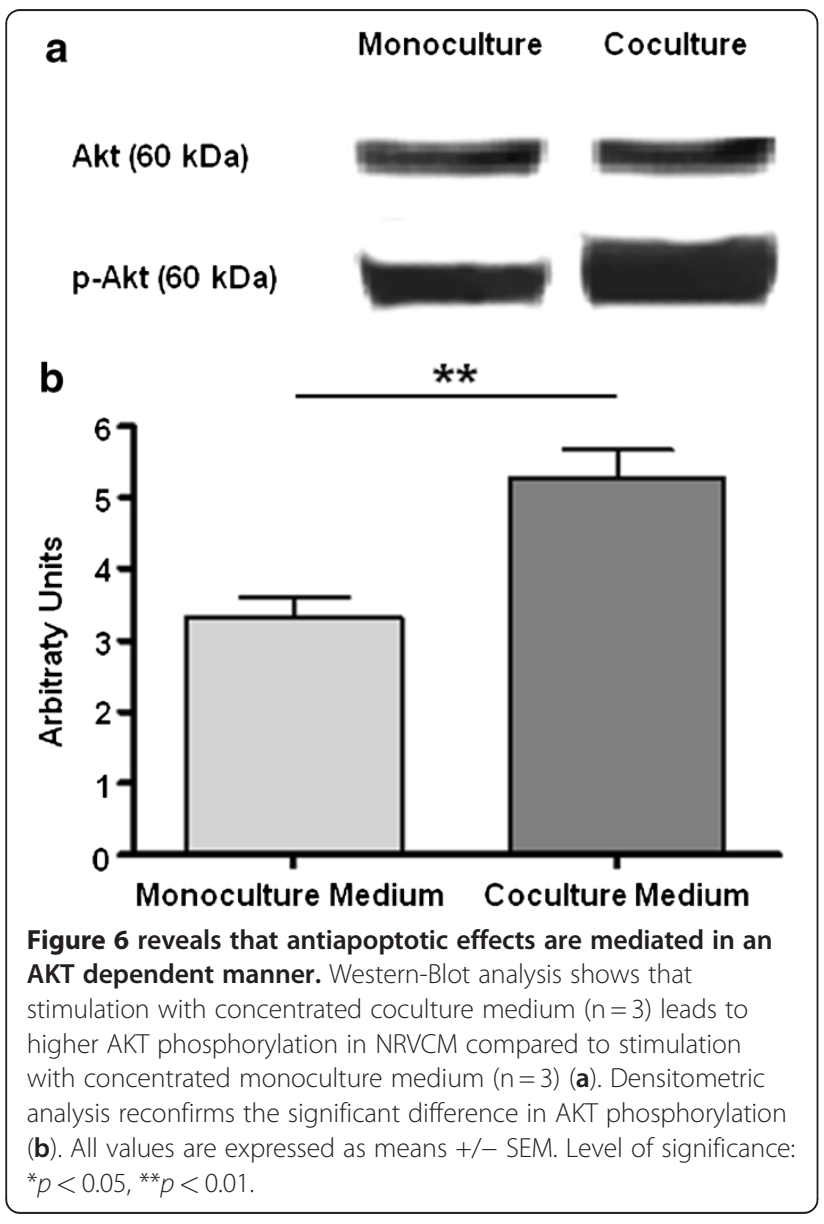

of some of these factors. We here demonstrate that cell-cell interaction with NRVCM indeed results in an upregulation of secretable antiapoptotic proteins in hematopoietic SC which in turn improved NRVCM survival by paracrine pathways.

We used a genomic approach to identify genes overexpressed in hematopoietic SC after coculture with NRVCM. In the search of factors with therapeutic potential after myocardial infarction we restricted our analysis to genes that are not only significantly upregulated but also encoded for secreted or membrane-bound proteins. Moreover, we finally selected proteins that are also known to interfere with antiapoptotic signaling. Using such a systematic approach we were able to narrow down an initial list of 3014 differentially regulated genes to 8 genes. Biological validation of our microarray data by Real-Time PCR confirmed significant results for CCL-12, MIF, FN-1 and GJA-5, while there was only a strong trend towards upregulation for CXCL1, CCL 6, EGFL 7 and TIMP. Hence, our results demonstrate that coculture with NRVCM leads to a change in the secretome of hematopoietic SC.

Chemokine ligand 12 (CCL-12) is a small cytokine belonging to the $\mathrm{C}-\mathrm{C}$ motif chemokine family. It is also known as monocyte chemotactic protein 5 (MCP-5) or as MCP-1 related chemokine. CCL-12 has been previously shown to be constitutively expressed in lymph nodes and thymus under basal conditions [24,25]. CCR2 is the receptor for CCL-12, which is not only expressed on monocytes and activated lymphocytes, but can also be found on cardiomyocytes [26]. Several experimental studies in rat neurons and murine lymphocytes indicated 
that stimulation of CCR2 can result in PI3K-dependent phosphorylation of AKT [27]. Thus, the increased phosphorylation of AKT observed in cocultured NRVCM may at least in part be explained by the paracrine action of CCL-12.

Another interesting candidate found upregulated in our experiments is the macrophage migration inhibitory factor (MIF). MIF is a pleiotropic cytokine which regulates the release of other pro-inflammatory cytokines and therefore is of great importance in mediating inflammatory responses [28]. MIF is known to be expressed and released upon stimulation from preformed storage pools by several cell types, including macrophages/monocytes, vascular smooth muscle cells and cardiomyocytes [29-31]. It has been shown that signal transduction of extracellular MIF involves a receptor tyrosine kinase (RTK)-like complex [32], promoting the activation of the PI3K/Akt pathway and cellular survival [22]. The biological relevance of this pathway has also been shown in the heart. Miller et al. demonstrated that MIF protects the rat heart from ischemia-reperfusion injury by stimulation of AMP-activated protein kinase (AMPK) in an autocrine manner [33]. Taken together, our results indicate that coculture of hematopoietic SC with NRVCM lead to an enhanced secretion of MIF by $\mathrm{SC}$ which may in turn protect NRVCM from apoptosis via phosphorylation of Akt in a paracrine manner.

Furthermore, it has become increasingly clear that interactions between extracellular matrix proteins and integrins not only mediate cell adhesion but also generate signals that play an important role in promoting cell survival [34]. Of note, Fibronectin (FN), one of the extracellular adhesive glycoproteins involved in these processes, was also found to be differentially regulated in our coculture experiments. FN is considered to provide survival signals for many cell types through its RGD motif that predominantly interacts with $\beta_{1}$-integrins, including $\alpha_{3} \beta_{1}$ and $\alpha_{5} \beta_{1}$ integrins [35]. The latter integrin was identified as an important regulator of apoptosis and is also expressed in cardiomyocytes [36-38]. Moreover, it has been repeatedly demonstrated that downstream signaling of $\alpha_{5} \beta_{1}$ integrin involves a PI3K-dependent phosphorylation of AKT $[23,39]$. We therefore hypothesize that coculture-induced overexpression of FN in hematopoietic $\mathrm{SC}$ also contributes to the protection of NRVCM against proapoptotic stimuli by an integrin mediated activation of the PI3K/AKT pathway.

Finally, our results demonstrated that Gap junction protein-5 alpha (GJA-5) is significantly upregulated in hematopoietic SC after coculture with NRVCM. GJA-5, which is also known as Connexin 40, plays an important role in the formation of gap junctions that are clusters of intercellular channels consisting of a hexameric assembly of proteins. These gap junction channels can link neighboring cells and thereby provide the molecular framework of intercellular communication [40]. Moreover, it has been shown that in a variety of cells overexpression of Connexin 40 leads to increased resistance against several types of injury including calcium overload and oxidative stress. Surprisingly, the protective activity of connexin proteins was found to be independent of gap junction channel function [41]. Our finding of GJA-5 overexpression in conditioned SC may thus also contribute to improved intercellular connections between cocultured cells and/or increased resistance against cellular injury.

Thus, at least to our knowledge, we here demonstrate for the first time a systematic analysis of the differential gene expression in adult hematopoietic SC modified by a coculture with NRVCM. Our results show that a direct interaction of hematopoietic SC with NRVCM leads to an overexpression of antiapoptotic proteins in adult hematopoietic SC which may improve NRVCM survival in a paracrine manner. These data are in line with previous reports. The Dzau group showed that cell culture medium conditioned by hypoxic mesenchymal SC that were transduced with an Akt-1 overexpressing retrovirus reduced apoptosis and necrosis in isolated NRVCM [42]. As the putative underlying mechanism the same group presented data that revealed upregulation of potentially cytoprotective molecules such as VEGF, FGF, HGF or IGF in hypoxic and genetically modified mesenchymal SC [11]. Other groups have confirmed the paracrine effects of bone marrow derived SC on ischemic CM $[43,44]$. Uemura et al. demonstrated that hypoxia induced apoptosis of CM could be reduced by coculture with bone marrow derived mesenchymal SC. Since VEGF, bFGF, IGF and SDF-1 were found in the supernatant of mesenchymal SC culture, this group also argued for a paracrine mediated effect [45]. Similar results were also reported in a clinical setting. KorfKlingebiel et al. used blood samples from 15 patients with acute myocardial infarction and isolated unselected nucleated bone marrow cells (BMSCs) and peripheral blood leukocytes (PBL). Cells were cultured to obtain conditioned supernatants. Cell culture medium of both cell types synergistically induced angiogenesis in a mouse aortic ring assay and protected rat CM from cell death induced by ischaemia followed by reperfusion [46].

How do our results fit into this context? We believe that our experimental approach is closer to the clinical setting, where bone marrow cells may directly interact with $\mathrm{CM}$. While the above cited papers examined the generation of cytoprotective factors in various types of SC under monoculture conditions, we developed a coculture model that gave us the possibility to systematically analyze the differential gene expression in hematopoietic $\mathrm{SC}$ modified by direct interaction with NRVCM. We and 
others have shown that systemically infused or intramyocardially injected hematopoietic SC are to some extent retained in the ischemic myocardium [14,47]. Based on the results presented in this study we propose that a direct interaction with NRVCM induces the expression of antiapoptotic proteins in myocardially retained hematopoietic SC which in turn may contribute to the beneficial effects of cellular cardiomyoplasty. Of note, we also found several genes differentially regulated that play a role in other potentially cardioprotective pathways such as angiogenesis and immune response. Thus, it seems likely that in vivo additional mechanisms contribute to the effects of hematopoetic stem cells.

Some limitations of our work need further appreciation. For several reasons we preferred a genomic approach to systematically analyze the effects of coculture on adult hematopoietic SC. Compared to a proteomic approach a genomic approach is technically less demanding, more comprehensive and offers the possibility to detect new genes with previously unknown effects in SC therapy. On the other hand, a genomic approach can overlook important posttranscriptional events such as alternative splicing. Furthermore, it was beyond the scope of this study to analyze the therapeutic potentials of novel cytoprotective factors. Further studies will be needed to determine the therapeutic potentials of overexpressing factors such as CCL-12, MIF, FN or GJA-5. Finally, despite the high degree of purification of hematopoetic stem cells in our experimental setting, we cannot exclude that other cell types, e.g. macrophages, also contribute to the observed effects.

\section{Conclusion}

In summary, we here present for the first time a comprehensive analysis of the gene expression program of hematopoietic SC after coculture with NRVCM. Thereby we were able to demonstrate that the direct interaction of hematopoietic SC with NRVCM leads to an upregulation of secretable and/or membrane bound antiapoptotic proteins which in turn improve NRVCM survival in a paracrine manner. Further studies will have to show whether the use of single cytoprotective factors can achieve similar results compared to cellular therapy after myocardial infarction.

\section{Additional files}

Additional file 1: Processing of conditioned SC, NRVCM and supernatants is described in the supplementary methods section.

Additional file 2: Table S1. Additional differentially regulated genes in cocultured hematopoietic SC listed according to their potential protective biological function.

\section{Competing interests}

The authors declare that they have no competing interests.

\section{Author details}

'Department of Internal Medicine III (Cardiology and Angiology), University Medical Center Schleswig-Holstein, Campus Kiel, Schittenhelmstr.12, D-24105, Kiel, Germany. ${ }^{2}$ Department of Internal Medicine III, University of Heidelberg, Heidelberg, Germany. ${ }^{3}$ Department of Internal Medicine V, University of Heidelberg, Heidelberg, Germany.

\section{Authors' contributions}

MR participated in conception and design of the study, collected and assemblied study material or patients, analyzed and interpreted data and drafted the manuscript. ML and CK participated in conception and design of the study, collected and assemblied study material or patients, analyzed and interpreted data. RW and VE also helped in the analysis and interpretation of experimental data. JK participated in the Collection/Assembly of study material or patients. HK participated in the design of the study and helped to draft the manuscript. Finally NF also helped to conceive the study design, analyzed and interpreted data and drafted the manuscript. All authors read and approved the final manuscript.

\section{Funding}

This work was supported by internal funds of the University of Heidelberg and Kiel.

Received: 22 September 2011 Accepted: 6 June 2012

Published: 6 June 2012

\section{References}

1. MacLellan WR, Schneider MD: Genetic dissection of cardiac control pathways. Annu Rev Physiol 2000, 62:289-319.

2. Beltrami AP, Barlucchi L, Torella D, Baker M, Limana F, Chimenti S, Kasahara H, Rota M, Musso E, Urbanek K, Leri A, Kajstura J, Nadal-Ginard B: Anversa: Adult cardiac stem cells are mutipotent and support myocardial regeneration. Cell 2003, 114:763-776.

3. Jackson K, Majka SM, Wang H, Pocius J, Hartley CJ, Majesky MW, Entman ML, Michael LH, Hirschi KK, Goodell MA: Regeneration of ischemic cardiac muscle and vascular endothelium by adult stem cells. J Clin Invest 2001, 107:1395-1402.

4. Murry CE, Reinecke H, Pabon LM: Regeneration gaps: observations on stem cells and cardiac repair. J Am Coll Cardiol 2006, 47:1777-1785.

5. Orlic D, Kajstura J, Chimenti S, Jakoniuk I, Anderson SM, Li B, Pickel J, McKay R, Nadal-Ginard B, Bodine DM, Leri A, Anversa P: Bone marrow cells regenerate infarcted myocardium. Nature 2001, 410:701-705.

6. Murry CE, Soonpaa MH, Reinecke H, Nakajima H, Rubart M, Pasumarthi KB, Virag Jl, Bartelmez SH, Poppa V, Bradford G, Dowell JD, Williams DA, Field LJ: Haematopoietic stem cells do not transdifferentiate into cardiac myocytes in myocardial infarcts. Nature 2004, 428:664-668.

7. Balsam LB, Wagers AJ, Christensen JL, Kofidis T, Weissman IL, Robbins RC: Haematopoietic stem cells adopt mature haematopoietic fates in ischemic myocardium. Nature 2004, 428:668-673.

8. Kinnaird T, Stabile E, Burnett MS, Lee CW, Barr S, Fuchs S, Eppstein SE: Marrow-derived stromal cells express genes encoding a broad spectrum of arteriogenic cytokines and promote in vitro and in vivo arteriogenesis through paracrine mechanisms. Circ Res 2004, 94:678-685.

9. Yoon YS, Wecker A, Heyd L, Park JS, Tkebuchava T, Kusano K, Hanley A, Scadova H, Qin G, Cha DH, Johnson KL, Aikawa R, Asahara T, Losordo DW: Clonally expanded novel multipotent stem cells from human bone marrow regenerate myocardium after myocardial infarction. J Clin Invest 2005, 115:326-338.

10. Nagaya N, Kangawa K, Itoh T, Iwase T, Murakami S, Miyahara Y, Fujii T, Uematsu M, Ohgushi H, Yamagishi M, Tokudome T, Mori H, Miyatake K, Kitamura S: Transplantation of mesenchymal stem cells improves cardiac function in a rat model of dilated cardiomyopathy. Circulation 2005, 112:1128-1135.

11. Gnecchi M, He H, Noiseux N, Liang OD, Zhang L, Morello F, Mu H, Melo LG, Pratt RE, Ingwall JS, Dzau VJ: Evidence supporting paracrine hypothesis for Akt-modified mesenchymal stem cell-mediated cardiac protection and functional improvement. FASEB J 2006, 20:661-669.

12. Kuhn C, Frank D, Will R, Jaschinski C, Frauen R, Katus HA, Frey N: DYRK1A is a novel negative regulator of cardiomyocyte hypertrophy. $J$ Biol Chem 2009, 284:17320-17327. 
13. Frank D, Kuhn C, van Eickels M, Gehring D, Hanselmann C, Lippl S, Will R, Katus HA, Frey N: Calsarcin-1 protects against angiotensin-II induced cardiac hypertrophy. Circulation 2007, 116:2587-2596.

14. Lutz M, Rosenberg M, Kiessling F, Eckstein V, Heger T, Krebs J, Ho AD, Katus $H A$, Frey N: Local injection of stem cell factor (SCF) improves myocardial homing of systemically delivered c-kit 1 bone marrow-derived stem cells. Cardiovasc Res 2008, 77:143-150

15. DeGeorge BR, Rosenberg M, Eckstein V, Gao E, Herzog N, Katus HA, Koch WJ, Frey N, Most P: BMP-2 and FGF-2 Synergistically Facilitate Adoption of a Cardiac Phenotype in Somatic Bone Marrow c-kit+/Sca-1+ Stem Cells. CTS 2008, 1:116-125.

16. Eberwine J, Yeh H, Miyashiro K, Cao Y, Nair S, Finnell R, Zettel M, Coleman P: Analysis of gene expression in single live neurons. Proc Natl Acad Sci USA 1992, 89:3010-3014.

17. Benjamini $Y$, Hochberg Y: Controlling the false discovery rate: a practical and powerful approach to multiple testing. Journal of the Royal Statistical Society, Series B (Methodological) 1995, 57:125-133.

18. Edgar R, Domrachev M, Lash AE: Gene Expression Omnibus: NCBI gene expression and hybridization array data repository. Nucleic Acids Res 2002, 30:207-210.

19. Huang DW, Sherman BT, Lempicki RA: Systematic and integrative analysis of large gene lists using DAVID Bioinformatics Resources. Nature Protoc 2009, 4:44-57.

20. Huang DW, Sherman BT, Lempicki RA: Bioinformatics enrichment tools: paths toward the comprehensive functional analysis of large gene lists. Nucleic Acids Res 2009, 37:1-13.

21. Gosselin RD, Varela C, Banisadr G, Mechighel P, Rostene W, Kitabgi P, MelikParsadaniantz S: Constitutive expression of CCR2 chemokine receptor and inhibition by MCP-1/CCL2 of GABA-induced currents in spinal cord neurones. J Neurochem 2005, 95:1023-1033.

22. Lue H, Thiele M, Franz J, Dahl E, Speckgens S, Leng L, Fingerle-Rowson G, Bucala R, Luscher B, Bernhagen J: Macrophage migration inhibitory factor (MIF) promotes cell survival by activation of the Akt pathway and role for CSN5/JAB1 in the control of autocrine MIF activity. Oncogene 2007, 26:5046-5059.

23. Fornaro M, Plescia J, Chheang S, Tallini G, Zhu YM, King M, Altieri DC, Languino LR: Fibronectin Protects Prostate Cancer Cells from Tumor Necrosis Factor-a-induced Apoptosis via the AKT/Survivin Pathway. J Biol Chem 2003, 278:50402-50411.

24. Sarafi M, Garcia-Zepeda E, MacLean J, Charo I, Luster A: Murine monocyte chemoattractant protein (MCP)-5: a novel CC chemokine that is a structural and functional homologue of human MCP-1. J Exp Med 1997, 185:99-109

25. Jia G, Gonzalo J, Lloyd C, Kremer L, Lu L, Martinez-A C, Wershil B, Gutierrez-Ramos J: Distinct expression and function of the novel mouse chemokine monocyte chemotactic protein-5 in lung allergic inflammation. J Exp Med 1996, 184:1939-1951.

26. Zhou L, Azfer A, Niu J, Graham S, Choudhury M, Adamski FM, Younce C, Binkley PF, Kolattukudy PE: Monocyte chemoattractant protein-1 induces a novel transcription factor that causes cardiac myocyte apoptosis and ventricular dysfunction. Circ Res 2006, 98:1177-1185

27. Webb A, Johnson A, Fortunato M, Platt A, Crabbe T, Christie MI, Watt GF, Ward SG, Jopling LA: Evidence for PI-3 K-dependent migration of Th17-polarized cells in response to CCR2 and CCR6 agonists. J Leukoc Biol 2008, 84:1202-1212.

28. Calandra T, Bernhagen J, Metz CN, Spiegel LA, Bacher M, Donnelly T, Cerami A, Bucala R: MIF as a glucocorticoid-induced modulator of cytokine production. Nature 1995, 377:68-71.

29. Calandra T, Bernhagen J, Mitchell RA, Bucala R: The macrophage is an important and previously unrecognized source of macrophage migration inhibitory factor. J Exp Med 1994, 179:1895-1902.

30. Burger-Kentischer A, Goebel H, Seiler R, Fraedrich G, Schaefer HE, Dimmeler $\mathrm{S}$, Kleemann R, Bernhagen J, Ihling C: Expression of macrophage migration inhibitory factor in different stages of human atherosclerosis. Circulation 2002, 105:1561-1566.

31. Willis MS, Carlson DL, Dimaio JM, White MD, White DJ, Adams GA 4th, Horton JW, Giroir BP: Macrophage migration inhibitory factor mediates late cardiac dysfunction after burn injury. Am J Physiol Heart Circ Physiol. 2005, 288:H795-H804.

32. Zernecke A, Bernhagen J, Weber C: Macrophage migration inhibitory factor in cardiovascular disease. Circulation 2008, 117:1594-1602.
33. Miller EJ, Li J, Leng L, McDonald C, Atsumi T, Bucala R, Young LH: Macrophage migration inhibitory factor stimulates AMP-activated protein kinase in the ischaemic heart. Nature 2008, 451:578-582.

34. Frisch SM, Ruoslahti E: Integrins and anoikis. Curr Opin Cell Biol 1997, 9:701-706

35. Kapila YL, Wang S, Johnson PW: Mutations in the heparin binding domain of fibronectin in cooperation with the $V$ region induce decreases in pp 125 (FAK) levels plus proteoglycan-mediated apoptosis via caspases. J Biol Chem 1999, 274:30906-30913.

36. Zhang Z, Vuori K, Wang H, Reed JC, Ruoslahti E: Integrin activation by R-ras. Cell 1996, 85:61-69.

37. O'Brien V, Frisch SM, Juliano RL: Expression of the integrin alpha 5 subunit in HT29 colon carcinoma cells suppresses apoptosis triggered by serum deprivation. Exp Cell Res 1996, 224:208-213.

38. Wu X, Sun Z, Foskett A, Trzeciakowski JP, Meininger GA, Muthuchamy M: Cardiomyocyte contractile status is associated with differences in fibronectin and integrin interactions. Am J Physiol Heart Circ Physiol 2010, 298:H2071-H2081

39. Gu J, Fujibayashi A, Yamada KM, Sekiguchi K: Laminin-10/11 and Fibronectin Differentially Prevent Apoptosis Induced by Serum Removal via Phosphatidylinositol 3-Kinase/Akt- and MEK1/ERK-dependent Pathways. J Biol Chem 2002, 277:19922-19928.

40. Kumar NM, Gilula NB: The gap junction communication channel. Cell 1996, 84:381-388.

41. Lin JH, Yang J, Liu S, Takano T, Wang X, Gao Q, Willecke K, Nedergaard M: Connexin mediates gap junction-independent resistance to cellular injury. J Neurosci 2003, 23:430-441.

42. Gnecchi M, He H, Liang OD, Melo LG, Morello F, Mu H, Noiseux N, Zhang L, Pratt RE, Ingwall JS, Dzau VJ: Paracrine action accounts for marked protection of ischemic heart by Akt-modified mesenchymal stem cells. Nat Med 2005, 11:367-368.

43. Takahashi M, Li TS, Suzuki R, Kobayashi T, Ito H, Ikeda Y, Matsuzaki M, Hamano K: Cytokines produced by bone marrow cells can contribute to functional improvement of the infarcted heart by protecting cardiomyocytes from ischemic injury. Am J Physiol Heart Circ Physiol 2006, 291:H886-H893.

44. Xu M, Uemura R, Dai $Y$, Wang $Y$, Pasha Z, Ashraf $M$ : In vitro and in vivo effects of bone marrow stem cells on cardiac structure and function. J Mol Cell Cardiol 2007, 42:441-448.

45. Uemura R, Xu M, Ahmad N, Ashraf M: Bone marrow stem cells prevent left ventricular remodelling of ischemic heart through paracrine signalling. Circ Res 2006, 98:1414-1421.

46. Korf-Klingebiel M, Kempf T, Sauer T, Brinkmann E, Fischer P, Meyer GP, Ganser A, Drexler H, Wollert KC: Bone marrow cells are a rich source of growth factors and cytokines: implications for cell therapy trials after myocardial infarction. Eur Hear J 2008, 29:2851-2858.

47. Cho HJ, Lee N, Lee JY, Choi YJ, Li M, Wecker A, Jeong JO, Curry C, Qin G, Yoon YS: Role of host tissues for sustained humoral effects after endothelial progenitor cell transplantation into the ischemic heart. J Exp Med 2007, 204:3257-3269.

doi:10.1186/1479-5876-10-115

Cite this article as: Rosenberg et al:: Coculture with hematopoietic stem cells protects cardiomyocytes against apoptosis via paracrine activation of AKT. Journal of Translational Medicine 2012 10:115.

\section{Submit your next manuscript to BioMed Central and take full advantage of:}

- Convenient online submission

- Thorough peer review

- No space constraints or color figure charges

- Immediate publication on acceptance

- Inclusion in PubMed, CAS, Scopus and Google Scholar

- Research which is freely available for redistribution 